PAPER

\section{Synthesis, characterization and applications of maghemite beads functionalized with rabbit antibodies}

To cite this article: A F R Rodriguez et al 2018 Nanotechnology 29365701

View the article online for updates and enhancements.

\section{Related content}

- $\frac{\text { Highly fluorescent and superparamagnetic }}{\text { nanosystem for biomedical applications }}$
Mariana P Cabrera, Paulo E Cabral Filho,
Camila M C M Silva et al.
- Versatile theranostics agents designed by
$\frac{\text { coating ferrite nanoparticles with }}{\text { biocompatible polymers }}$
M Zahraei, M Marciello, A Lazaro-Carrillo
et al.
- Synthesis, characterization and in vitro
evaluation of exquisite targeting
SPIONs-PEG-HER in HER2+ human
breast cancer cells
Javad Hamzehalipour Almaki, Rozita
Nasiri, Ani Idris et al.

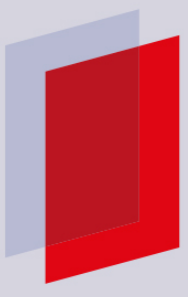

\section{IOP ebooks'}

Bringing you innovative digital publishing with leading voices to create your essential collection of books in STEM research. Start exploring the collection - download the first chapter of every title for free. 


\title{
Synthesis, characterization and applications of maghemite beads functionalized with rabbit antibodies
}

\author{
A F R Rodriguez ${ }^{1,6}$ (1), C O Rocha ${ }^{5}$, R D Piazza ${ }^{5}$ C C dos Santos ${ }^{5}$, \\ M A Morales ${ }^{2}$, F S E D V Faria ${ }^{1}$, M Zubair Iqbal ${ }^{3}$, L Barbosa ${ }^{4}$, Y O Chaves ${ }^{4}$, \\ L A Mariuba ${ }^{4}$, M Jafelicci $\mathrm{Jr}^{5}$ and R F C Marques ${ }^{5}$ \\ ${ }^{1}$ Federal University of Acre, Laboratory of Nanobiotechnology, Rio Branco, 69920-900, AC, Brazil \\ ${ }^{2}$ Federal University of Rio Grande do Norte, Department of Theoretical and Experimental Physics, Natal, \\ 59078-970, RN, Brazil \\ ${ }^{3}$ Division of Functional Materials and Nano-Devices, Ningbo Institute of Materials Technology \& \\ Engineering, Chinese Academy of Sciences, People's Republic of China \\ ${ }^{4}$ Instituto Leonidas e Maria Deane, Fundação Oswaldo Cruz, Adrianópolis, 69057-070, AM, Brazil \\ ${ }^{5}$ São Paulo State University-UNESP, Laboratory of Magnetic Materials and Colloids, Institute of \\ Chemistry, Caixa postal 355, Araraquara 14800-900, SP, Brazil
}

E-mail: anselmorodriguez73@gmail.com

Received 23 January 2018, revised 4 June 2018

Accepted for publication 12 June 2018

Published 28 June 2018

\begin{abstract}
Magnetic nanoparticles (NPs) have attracted great attention owing to their applications in the biomedical field. In the present work, maghemite $\left(\gamma \mathrm{Fe}_{2} \mathrm{O}_{3}\right)$ NPs of $6.5 \mathrm{~nm}$ were prepared using a sonochemical method and used to prepare magnetic beads through silanization with 3-aminopropyltrimethoxysilane (APTS). Subsequently, amino groups in the resulting APTS $-\gamma \mathrm{Fe}_{2} \mathrm{O}_{3}$ beads were converted to carboxylic acid (CARB- $\gamma \mathrm{Fe}_{2} \mathrm{O}_{3}$ ) through the succinic anhydride reaction, as confirmed by transmission electron microscopy (TEM), Fourier transform infrared spectroscopy and dynamic light scattering (DLS) measurements. The size of these beads was measured as $12 \mathrm{~nm}$ and their hydrodynamic diameter as $490 \mathrm{~nm}$, using TEM analysis and DLS, respectively. The CARB- $\gamma \mathrm{Fe}_{2} \mathrm{O}_{3}$ beads were further functionalized by immobilizing rabbit antibodies on their surfaces; the immobilization was confirmed by flow cytometry and ionic strength. The samples were further characterized by Mössbauer spectroscopy and DC magnetization measurements. Studies on magnetic relaxivities showed that magnetic beads present great potential for application in MR imaging.
\end{abstract}

Keywords: magnetic nanobeads, flow cytometry, MRI, mössbauer spectroscopy, XRD, rabbit antibody

(Some figures may appear in colour only in the online journal)

\section{Introduction}

During the last few decades, researchers have been developing methods for the immobilization of proteins and enzymes on magnetic nanoparticles (NPs) [1], useful for magnetic resonance imaging (MRI) [2]. Various methods have been employed to synthesize iron oxide magnetic NPs, among the

\footnotetext{
${ }^{6}$ Author to whom any correspondence should be addressed.
}

most common being co-precipitation [3], sol-gel [4], solid state [5], sonochemical [6], microwave assisted [7] and thermal decomposition [8]. These solution based methods allow the production of NPs with controllable size, morphology and crystallinity, and variable surface chemistry. Their biocompatibility and appropriate functionalization are key conditions for their use in biomedicine [9]. Magnetic iron oxide NPs show superparamagnetic behavior, very low coercivity and high magnetic moment per gram of sample. To 
provide stability in water, iron oxide NPs are usually capped with a variety of functional groups such as amino (-NH2) and carboxylic groups (-COOH). Magnetic beads are small particles consisting of a non-magnetic matrix-e.g. silica or polystyrene-with embedded ferromagnetic NPs [10]. This allows the manipulation of beads through magnetic forces, but avoids agglomeration of the beads since no permanent magnetic moment remains after removing an external magnetic field.

A wide selection of silica-coated magnetic NPs can be used as substrates for the attachment, through polar interactions, of a broad range of biomolecules. However, proteins do not normally bind effectively to silica beads; thus, a simple treatment to have more reactive surface groups, such as carboxylic groups (-COOH) [11] will greatly enhance protein binding.

The large surface-to-volume ratio of magnetic beads provides a further use for chemical bonding of target macromolecules [12]. Magnetic beads are well established for manipulation of biomolecules, such as proteins, cells, or bacteria [13], and can be applied in magnetic bioseparations [14]. Magnetic beads may also serve as contrast enhancing agents for MRI, for releasing heat upon absorption of radio frequencies, or they may be used for magnetic drug targeting $[15,16]$.

In this work, we will summarize a chemical route for the synthesis of magnetic beads using APTS and maghemite. Modification of the bead's surface was performed by succinic anhydride reaction. We have used rabbit antibodies to prove the ability of magnetic beads to couple proteins on their surface. The physical and chemical properties of magnetic beads were studied using Fourier transform IR spectroscopy (FTIR), zeta potential, transmission electron microscopy (TEM), x-ray diffraction analysis (XRD), dynamic light scattering (DLS), DC magnetization, Mössbauer spectroscopy and flow cytometry. Finally, we will apply the beads in various aqueous concentrations for MRI and in high-resolution molecular imaging.

\section{Experimental}

\subsection{Materials}

All chemicals were used as received from suppliers:

Iron (II) chloride tetrahydrate (97\%), succinic anhydride (99.0\%) and 3-aminopropyltrimethoxysilane (APTS) (98.0\%) were purchased from Sigma-Aldrich Brazil. Dimethylformamide (DMF, 99.9\%) was acquired from Panreac. Sodium hydroxide $(\mathrm{NaOH}, 97 \%)$ and ammonium hydroxide $\left(\mathrm{NH}_{4} \mathrm{OH}\right.$, $30 \%(\mathrm{v} / \mathrm{v}))$ were purchased from Synth. Iron (III) chloride hexahydrate and sodium chloride $(99.0 \%)$ were purchased from Mallinckrodt Chemicals.

\subsection{Synthesis of magnetic $\mathrm{NPs}_{(}\left(\mathrm{Fe}_{2} \mathrm{O}_{3}\right)$ and beads with amine groups (APTS- $\gamma \mathrm{Fe}_{2} \mathrm{O}_{3}$ )}

Magnetite was synthesized by sonoprecipitation method [17]. The NPs were obtained by dissolving $1.019 \mathrm{~g}$ of $\mathrm{FeCl}_{2} \cdot 4 \mathrm{H}_{2} \mathrm{O}$ and $2.773 \mathrm{~g}$ of $\mathrm{FeCl}_{3} \cdot 6 \mathrm{H}_{2} \mathrm{O}$ (in a molar ratiol:2) in $80 \mathrm{ml}$ of

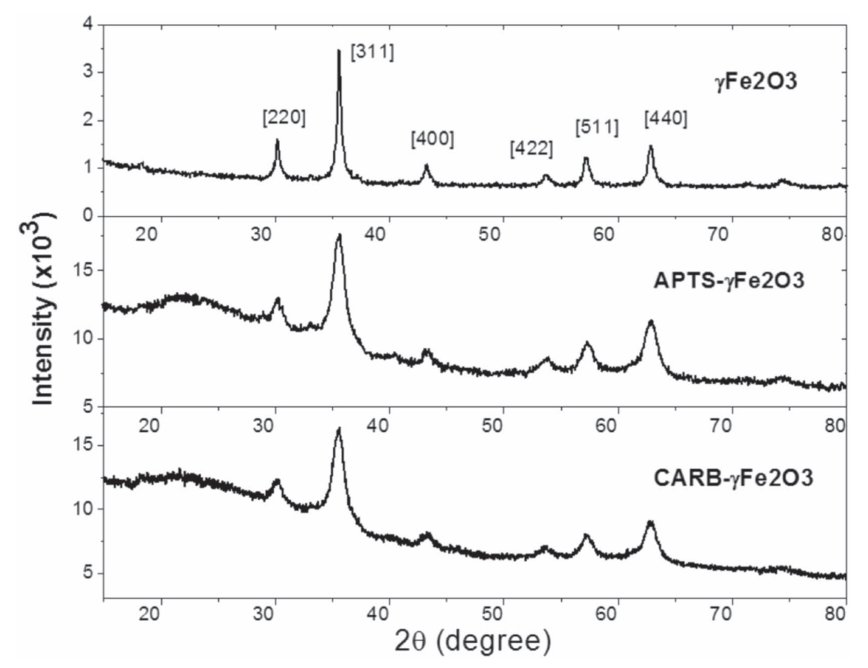

Figure 1. Diffraction patterns of bare iron oxide $\gamma \mathrm{Fe}_{2} \mathrm{O}_{3}$, APTS $-\gamma \mathrm{Fe}_{2} \mathrm{O}_{3}$ and CARB $-\gamma \mathrm{Fe}_{2} \mathrm{O}_{3}$ beads. Numbers are the Miller indices.

distilled water. An aqueous solution at $80{ }^{\circ} \mathrm{C}$ was prepared with sodium hydroxide and $1.0 \mathrm{ml}$ aliquots of $\mathrm{H}_{2} \mathrm{O}$ added until the solution reached $\mathrm{pH} 12$. Then $1.6 \mathrm{ml}$ of the iron solution was added to the above alkaline solution. This solution was agitated with an ultrasonic tip probe for one hour, with $3 \mathrm{~s}$ of pulse on and $3 \mathrm{~s}$ of pulse off. Transformation of magnetite to maghemite occurred after exposing the aqueous suspension of NPs to air at room temperature for one week.

To prepare magnetic beads, we added $200 \mu \mathrm{l}$ of APTS to the above maghemite suspension. The as-produced beads were labeled as APTS $-\gamma \mathrm{Fe}_{2} \mathrm{O}_{3}$.

\subsection{Synthesis of beads with carboxylic groups (CARB- $\left.\gamma \mathrm{Fe}_{2} \mathrm{O}_{3}\right)$}

In a second step, $200 \mathrm{mg}$ of APTS- $\gamma \mathrm{Fe}_{2} \mathrm{O}_{3}$ beads was dispersed in $30 \mathrm{ml}$ of dimethylformamide (DMF). A solution of $0.3 \mathrm{~g}$ of succinic anhydride and $20 \mathrm{ml}$ of DMF was prepared. These solutions were mixed and placed in a distillation flask with bubbling argon at room temperature and for $8 \mathrm{~h} \mathrm{[18].}$

\subsection{Functionalization of magnetic beads with rabbit antibodies}

The functionalization of magnetic beads with rabbit antibodies was done by using the methodology reported by Greg [19] — briefly, $20 \mathrm{mg}$ of maghemite beads were washed three times with phosphate buffer $(0.01 \mathrm{M})$ and re-suspended in $1 \mathrm{ml}$ of ultrapure water. Then, $1 \mathrm{ml}$ of rabbit antibody at various concentrations $\left(10,8,5,2.5,2,1\right.$ and $\left.0.5 \mu \mathrm{g} \mathrm{ml}^{-1}\right)$, $400 \mu \mathrm{l}\left(0.54 \mathrm{mg} \mathrm{ml}^{-1}\right)$ EDAC (1-ethyl-3-(3-dimethylaminopropyl)carbodiimide, hydrochloride) and $5 \mathrm{mM}$ NHS (Nhydroxysuccinimide) were added to the magnetite dispersion. The beads were washed again, and the blocking buffer (1 M glycine at $\mathrm{pH}$ 8.0) was added and mixed for $1 \mathrm{~h}$. Then, fluorescent secondary antibody (Anti-rabbit Alexa 488) was added at the dilution of $1 / 200$, incubated for $30 \mathrm{~min}$, and washed twice with PBS. Finally, the magnetic beads were measured by flow cytometry (FACS Canto II). 

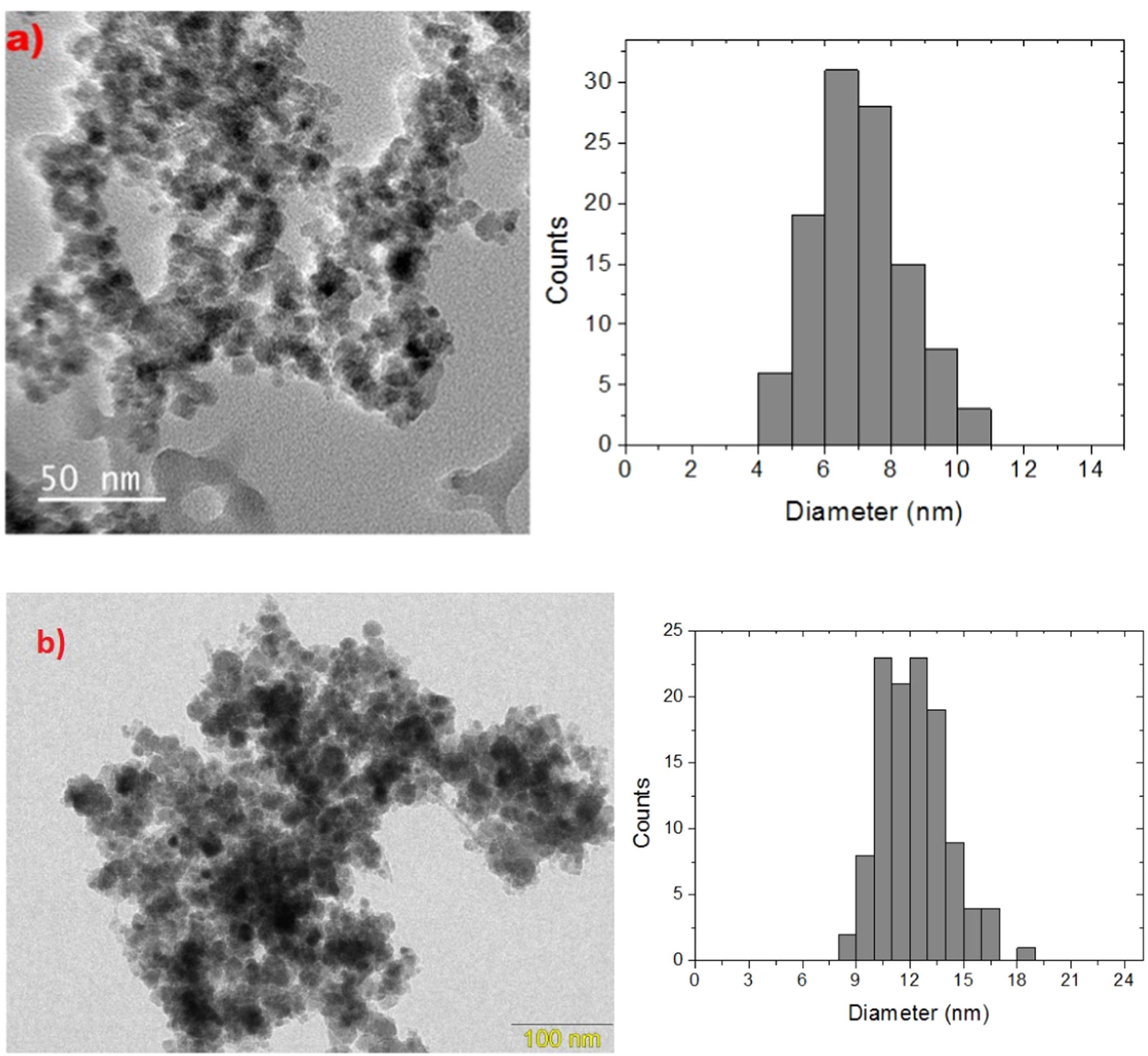

Figure 2. (a) TEM micrograph of bare $\gamma \mathrm{Fe}_{2} \mathrm{O}_{3}$ NPs, and their size distribution. (b) TEM micrograph of APTS- $\gamma \mathrm{Fe}_{2} \mathrm{O}_{3}$ beads, and their size distribution.

\subsection{XRD measurements}

Structural characterization of samples was carried out with XRD using a MiniFlexII Rigaku diffractometer equipped with a $\mathrm{CuK} \alpha$ radiation source. XRD data were collected in the $2 \theta$ range between $10^{\circ}$ and $85^{\circ}$ with a scan rate of $5^{\circ} / \mathrm{min}$ and steps of $0.02^{\circ}$.

\subsection{TEM}

A TEM micrograph was obtained on a Philips CM120 microscope. The beads were dispersed in isopropanol and one drop of the dispersion was deposited on a carbon covered copper grid.

\subsection{Infrared spectroscopy (FTIR)}

FTIR spectra were recorded in the reflectance mode on a Perkin-Elmer Frontier spectrometer to identify the composition of the NPs and the lipase content. The samples were milled, dispersed in $\mathrm{KBr}$ and pressed into pellets. Spectra were recorded in the range of $4000-400 \mathrm{~cm}^{-1}$ with a nominal resolution of $4.0 \mathrm{~cm}^{-1}$ and through 64 scans.

\subsection{Zeta potential and DLS measurements}

Zeta potential and the particles' hydrodynamic size were measured using a Zetasizer Nanoseries NanoZS, from Malvern Instruments. For Zeta potential measurements, the samples were previously suspended in $\mathrm{NaCl} 1 \mathrm{mmol} \mathrm{L}^{-1}$ 


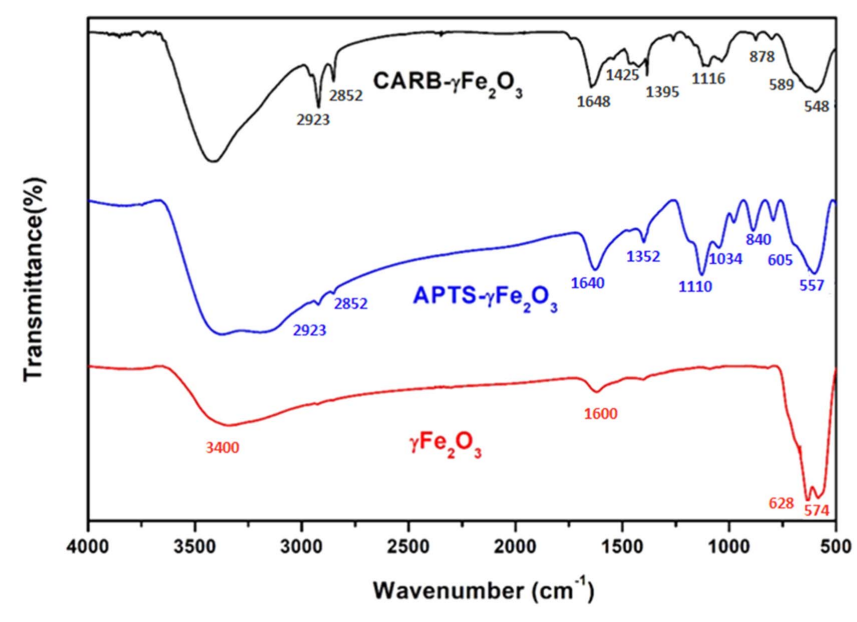

Figure 3. Infrared spectroscopy of $\gamma \mathrm{Fe}_{2} \mathrm{O}_{3}$, APTS- $\gamma \mathrm{Fe}_{2} \mathrm{O}_{3}$ and CARB- $\gamma \mathrm{Fe}_{2} \mathrm{O}_{3}$ samples.

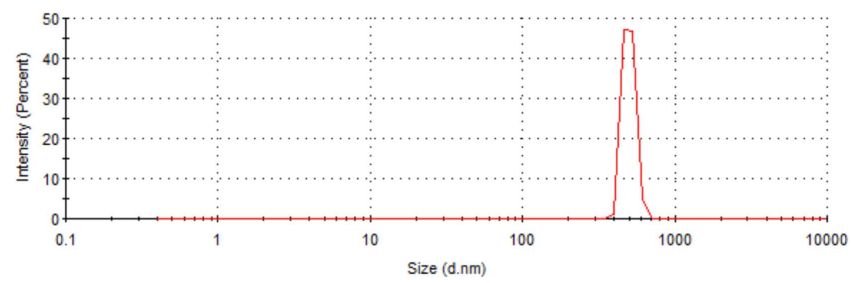

Figure 4. DLS of APTS $-\gamma \mathrm{Fe}_{2} \mathrm{O}_{3}$ NPs dispersed in water.

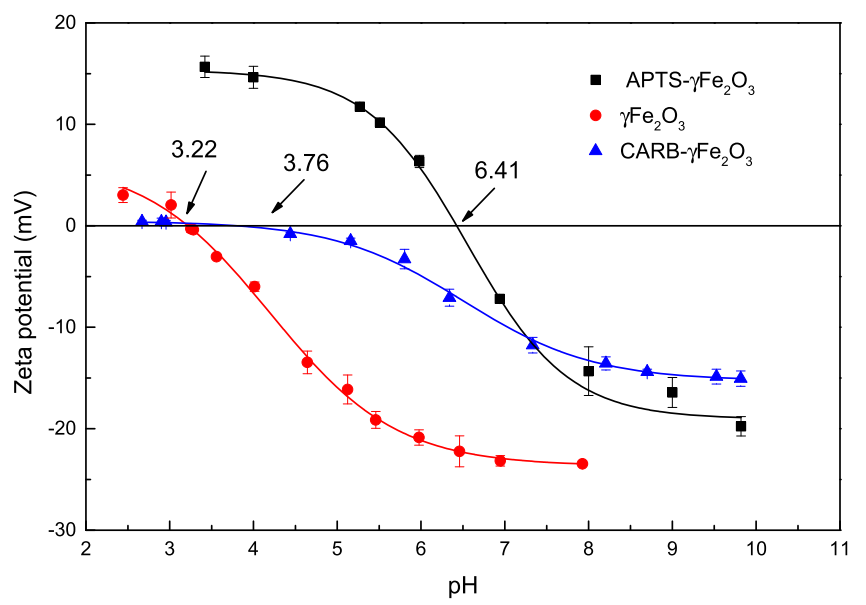

Figure 5. $\mathrm{pH}$ dependence of Zeta potential of $\gamma \mathrm{Fe}_{2} \mathrm{O}_{3}$, APTS$\gamma \mathrm{Fe}_{2} \mathrm{O}_{3}$ and CARB- $\gamma \mathrm{Fe}_{2} \mathrm{O}_{3}$ samples.

solution. The Zeta potential was evaluated in the $\mathrm{pH}$ range of 2-10 and the samples were titrated with $\mathrm{NaOH}$ and HCL $0.1 \mathrm{~mol} \mathrm{~L}^{-1}$ solution.

\subsection{Flow cytometry}

The samples were analyzed by FSC (Forware Scatter) size, SSC (Side Scatter) granularity and fluorescence in a flow cytometer (FACS Canto®II), and the results were analyzed using FlowJo® software version 9.3.2 of the Tree Star $(\subset$ with the flow cytometry platform at Fiocruz-ILMD.

\subsection{Mössbauer spectroscopy}

${ }^{57} \mathrm{Fe}$ Mossbauer spectra were recorded in the transmission mode at 300 and $12 \mathrm{~K}$ by using a spectrometer from SEECo equipped with a helium closed cycle cryostat from Janis; the gamma ray source had an activity of $25 \mu \mathrm{Ci}$. The spectra were fitted by using Normos 90 software; the reported isomer shifts are related to $\alpha-\mathrm{Fe}$ metal at room temperature.

\subsection{Magnetic characterization}

DC magnetization measurements were made in a physical properties measurement system, (PPMS-Dynacool from Quantum Design) equipped with a vibrating sample magnetometer (VSM). The magnetization as a function of temperature was determined in the zero field cooled (Mzfc) and field cooled (Mfc) modes under a magnetic field of $70 \mathrm{Oe}$; both measurements were made in the warming mode.

\subsection{2. $M R I$}

The transverse $\left(T_{2}\right)$ and longitudinal $\left(T_{1}\right)$ relaxation times and relaxivities $\left(r_{2}, r_{1}\right)$ of as-prepared NPs were performed on a 0.55 T MRI instrument (Shanghai Niumai Corporation Ration NM120-Analyst). Dilutions of iron oxide NPs samples in water with various concentrations $(0.08,0.18,0.36,0.72$ and $1.43 \mathrm{mM}$ ) were used for transverse and longitudinal relaxation times and $T_{2}$ imaging with additional concentrations (2.84 and $5.68 \mathrm{mM}$ ) as compared to water. Relaxation times $T_{2}$ and $T_{1}$ measurements were performed using TE (echo time) $=20 \mathrm{~ms}$ and TR (repetition time) $=4000 \mathrm{~ms}$. $T_{2}$-weighted MR images were performed on running a SE (spin-echo) sequence with $\mathrm{TR}=1600 \mathrm{~ms}$ and $\mathrm{TE}=20 \mathrm{~ms}$.

\section{Results and discussion}

\subsection{Structural characterization}

3.1.1. $X R D$. The patterns of bare maghemite particles and beads are shown in figure 1 . The diffractograms show peaks relative to the inverse spinel structure due to maghemite, no second phase like hematite or ferrihydrite was found. By using the Scherrer equation and the full width half maximum of the peak [311], [220], [511] and [440] we have determined the $\gamma \mathrm{Fe}_{2} \mathrm{O}_{3}$ particle size of $(6.9 \pm 0.9) \mathrm{nm}$, the samples APTS- $\gamma \mathrm{Fe}_{2} \mathrm{O}_{3}$ and CARB- $\gamma \mathrm{Fe}_{2} \mathrm{O}_{3}$ presented similar crystallite sizes to the bare $\gamma \mathrm{Fe}_{2} \mathrm{O}_{3} \mathrm{NPs}$, revealing that the procedure of coating with succinic anhydride and 3-aminopropyltrimethoxysilane do not affect the magnetic core. In the APTS- $\gamma \mathrm{Fe}_{2} \mathrm{O}_{3}$ and CARB- $\gamma \mathrm{Fe}_{2} \mathrm{O}_{3}$ patterns, the broad peak at about $22^{\circ}$ is related to the amorphous $\mathrm{SiO} 2$. The diffraction peaks in figure 1 are consistent with the standard structure of maghemite (JCPDS card No. 39-1346).

3.1.2. TEM analyses. A TEM image of bare $\gamma \mathrm{Fe}_{2} \mathrm{O}_{3}$ NPs is presented in figure 2(a) along with their size distribution; the NPs have a spherical morphology and are not aggregated. The size distribution has a mean diameter of $6.5 \mathrm{~nm}$. This result is 
A
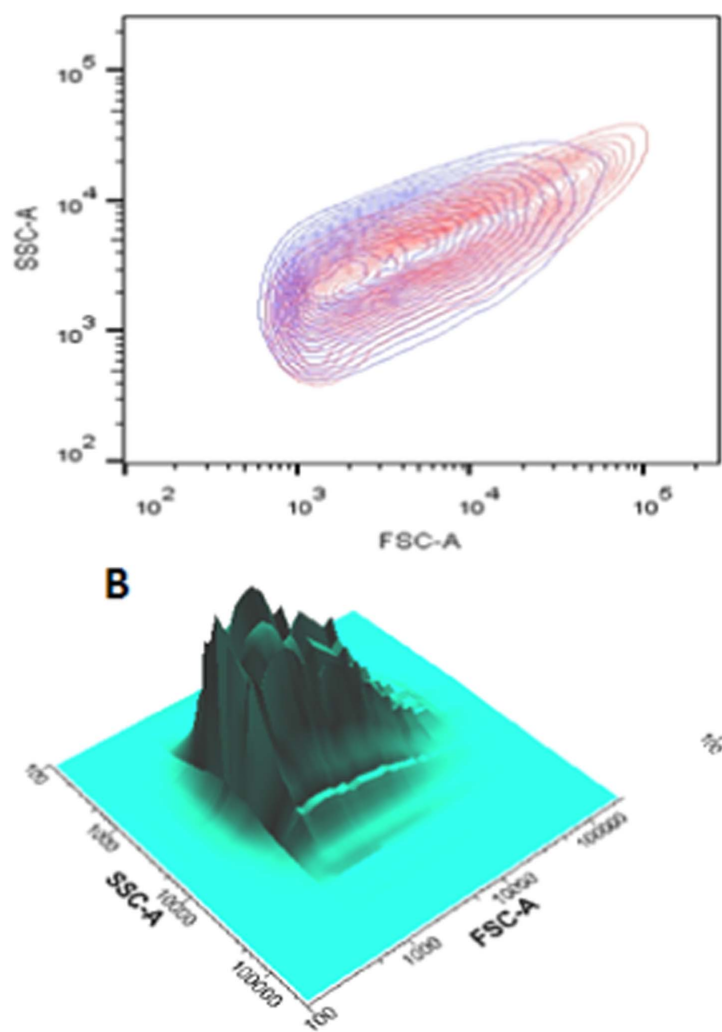

- CARB- $\gamma \mathrm{Fe}_{2} \mathrm{O}_{3}$

APTS- $\gamma \mathrm{Fe}_{2} \mathrm{O}_{3}$

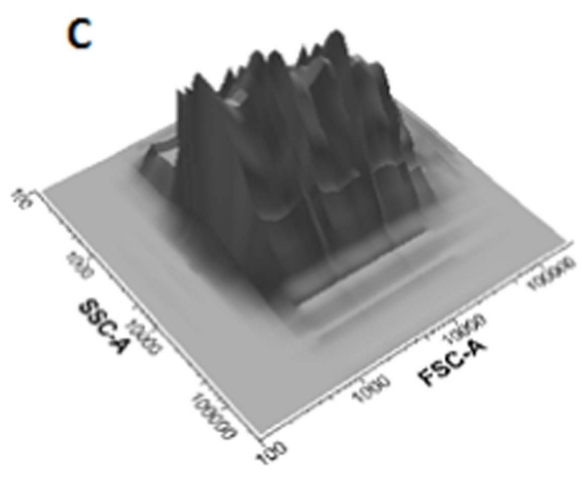

Figure 6. Overlap of APTS- $\gamma \mathrm{Fe}_{2} \mathrm{O}_{3}$ and CARB- $\gamma \mathrm{Fe}_{2} \mathrm{O}_{3}$ beads by FSC size and complexity SSC (A), surface chart of the correlation between FSC, SSC and the amount of NPs demonstrated by the APTS $-\gamma \mathrm{Fe}_{2} \mathrm{O}_{3}$ beads (B) and CARB- $\gamma \mathrm{Fe}_{2} \mathrm{O}_{3}$ beads (C).
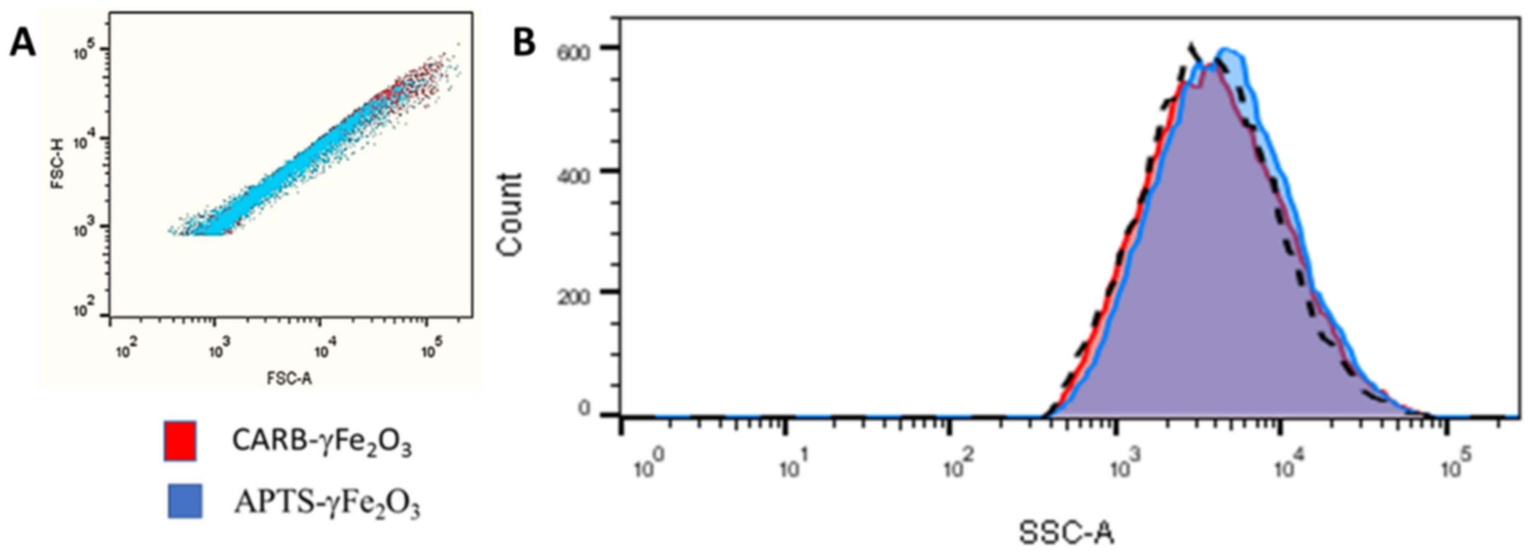

Figure 7. Evaluation of the uniformity of the samples correlating the size of the FSC-A area by the FSC-H height.

in close agreement with the crystallite size obtained in the XRD analysis.

A TEM image of APTS- $\gamma \mathrm{Fe}_{2} \mathrm{O}_{3}$ beads is presented in figure 2(b) along with their size distribution; the beads have a rounded morphology and form small agglomerates. The size distribution of the sample has a mean diameter of $12 \mathrm{~nm}$. This result confirms the $\mathrm{SiO}_{2}$ coating of the $\gamma \mathrm{Fe}_{2} \mathrm{O}_{3}$ NPs.

\subsection{Vibrational characterization of magnetic beads}

3.2.1. Infrared spectroscopy. The FTIR spectrum presented in figure 3 shows bands at 628 and $574 \mathrm{~cm}^{-1}$ which are assigned to the $\mathrm{Fe}-\mathrm{O}$ stretching vibration in $\gamma \mathrm{Fe}_{2} \mathrm{O}_{3}$, these bands appear slightly shifted in the spectra of the APTS- $\gamma \mathrm{Fe}_{2} \mathrm{O}_{3}$ and CARB- $\gamma \mathrm{Fe}_{2} \mathrm{O}_{3}$ samples. The bands at 3400 and $1600 \mathrm{~cm}^{-1}$ correspond to the $\mathrm{O}-\mathrm{H}$ stretching and bending vibrations of adsorbed water, respectively [20]. The spectrum of the APTS- $\gamma \mathrm{Fe}_{2} \mathrm{O}_{3}$ sample has absorption bands at $1034 \mathrm{~cm}^{-1}$ and $1110 \mathrm{~cm}^{-1}$ which correspond to $\mathrm{Si}-\mathrm{O}$ stretchings, confirming the silanization reaction. The amine group shows $\mathrm{N}-\mathrm{H}$ out-ofplane bending at $870 \mathrm{~cm}^{-1}$ and $\mathrm{C}-\mathrm{N}$ stretchings at $1352 \mathrm{~cm}^{-1}$. The asymmetric and symmetric absorption bands at 1648 and $1425 \mathrm{~cm}^{-1}$ correspond to carboxylate stretching vibrations, and confirm the functionalization with succinic anhydride. The bands located in between $1425-1380 \mathrm{~cm}^{-1}$ are a combination of $\mathrm{C}-\mathrm{O}$ stretchings and $\mathrm{O}-\mathrm{H}$ deformation vibration. The band at 


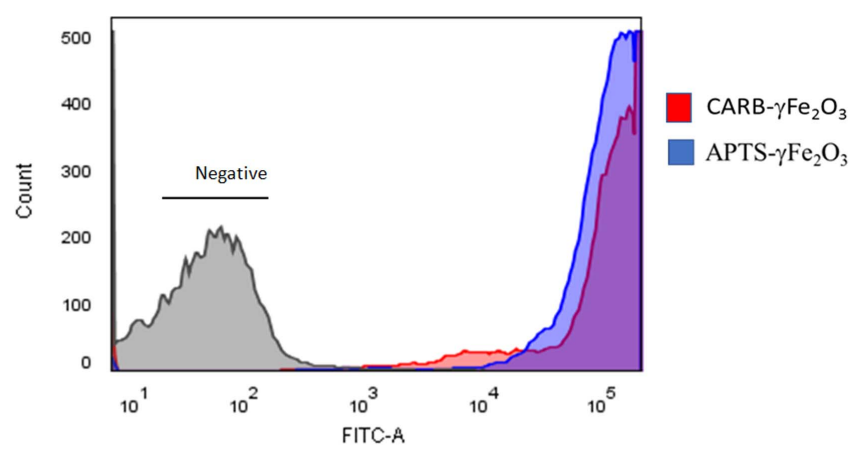

Figure 8. Overlays of the histograms showing the fluorescence of the secondary Alexa 488 Anti-Rabbit in the APTS- $\gamma \mathrm{Fe}_{2} \mathrm{O}_{3}$ and CARB $-\gamma \mathrm{Fe}_{2} \mathrm{O}_{3}$ beads conjugated in $10 \mu \mathrm{g}$ concentrations of primary rabbit.

$1264 \mathrm{~cm}^{-1}$ indicates the presence of carboxylic acid dimer [20]. Furthermore, the signals at $2923 \mathrm{~cm}^{-1}$ and $2852 \mathrm{~cm}^{-1}$, correspond to asymmetric and symmetric $\mathrm{C}-\mathrm{H}$ vibrations, these bands are also observed in $\mathrm{CARB}-\gamma \mathrm{Fe}_{2} \mathrm{O}_{3}$ and APTS $-\gamma \mathrm{Fe}_{2} \mathrm{O}_{3}$ samples.

3.2.2. DLS and zeta potential. Figure 4 shows the DLS measurement of APTS- $\gamma \mathrm{Fe}_{2} \mathrm{O}_{3}$ particles in water. It shows beads with hydrodynamic mean diameter of $490 \mathrm{~nm}$. Comparison of bead size from DLS and TEM techniques reveal aggregation of this sample.

In addition, the magnetic beads were evaluated by Zeta potential measurements. The isoelectric point (IEP) corresponds to the regime where the net charge on the NPs is zero. The zeta potentials as a function of $\mathrm{pH}$ curves are shown in figure 5. The IEP for $\gamma \mathrm{Fe}_{2} \mathrm{O}_{3} \mathrm{NPs}$ is located at $\mathrm{pH}=3.22$. It can be seen that the polycondensation with APTS shifted the IEP to 6.41, which corresponds to the APTS pKa value. Therefore, the APTS $-\gamma \mathrm{Fe}_{2} \mathrm{O}_{3}$ beads have a positive electrical potential at low $\mathrm{pH}(<6.41)$ and negative potential at high $\mathrm{pH}[21,22]$. Similar behavior is observed after functionalization of APTS- $\gamma \mathrm{Fe}_{2} \mathrm{O}_{3}$ beads with succinic anhydride. The $\mathrm{pKa}$ value of succinic acid is 4.2, while the IEP for CARB- $\gamma \mathrm{Fe}_{2} \mathrm{O}_{3}$ is at 3.76; this result confirms the functionalization of APTS $-\gamma \mathrm{Fe}_{2} \mathrm{O}_{3}$ with carboxylic groups.

\subsection{Morphometric analysis of beads (size and complexity)}

The morphometric characteristics of the NPs were analyzed by flow cytometry via the fluorescence detector parameters that measure the degree of direct scattering (FSC, $Y$-axis), and the degree of lateral dispersion or complexity-lateral dispersion (SSC, $X$-axis), and plotted on a logarithmic scale (figure 6(A)). The results show that the APTS $-\gamma \mathrm{Fe}_{2} \mathrm{O}_{3}$ beads were smaller in size than the CARB $-\gamma \mathrm{Fe}_{2} \mathrm{O}_{3}$ beads observed in 3D-size plots by complexity (figures 6(B), (C)).

3.3.1. Morphometric analysis of beads (uniformity). Although the initial morphometric characteristics showed that the particles had different sizes, it was verified that there was aggregation between the particles during the treatment; the singlet was analyzed by checking the particle uniformity in the parameters of lateral dispersion intensity (SSC-A) and uniformity (FSC-A) and (FSC-H). The results show that there is no difference between mean lateral dispersion intensity in both particle types compared to the untreated control (figure 7(B)) and that the particles follow a uniformity pattern (figure $7(\mathrm{~A})$ ) of $98.7 \%$ for APTS $-\gamma \mathrm{Fe}_{2} \mathrm{O}_{3}$ and $97.4 \%$ for CARB $-\gamma \mathrm{Fe}_{2} \mathrm{O}_{3}$.

Thus, we have performed several experiments to characterize the morphometric parameters of the beads essential to applications in biotechnology; the purity analysis can facilitate the identification of beads by flow cytometry.

\subsubsection{Evaluation of fluorescence coupling capacity in beads.}

The uniformity of the samples correlating the size of the FSCA area by the FSC-H height (see figure 8) was also evaluated, showing that both samples have approximately uniform sizes comprising $92.2 \%$ and $92.9 \%$ within the analysis range, respectively.

Two subpopulations of coupled NPs were analyzed, in order to determine the percentage of fluorescence coupling according to the types of pretreatment; these results are presented in table 1 .

The percentage of coupling is calculated by the initial analysis of the percentage of events within the gate determined by size and complexity (figure 6); after determining the area of analysis of the beads, the percentage of fluorescence is determined in relation to the beads without coupling or cut off. The fluorescence above the cut line determines the percentage of fluorescence (figure 8) calculated through the Flowjo program or by multiplying the fluorescence gate (fluorescence) mean by the gate (count) of size and complexity.

In the fluorescence analysis, the histograms show that both samples have about $95 \%$ coupling; this is confirmed in figure 8 by the overlapping histogram graph, that shows two populations of magnetic NPs: low coupling and high coupling (see table 1). ATPS $-\gamma \mathrm{Fe}_{2} \mathrm{O}_{3}$ magnetic beads exhibited larger populations with high coupling percentage (93.3\%) when compared to CARB- $\gamma \mathrm{Fe}_{2} \mathrm{O}_{3}$ magnetic beads (85\%) at either of the $10 \mu \mathrm{g}$ or $0.5 \mu \mathrm{g}$ of rabbit antibody concentrations shown in table 1 . However, no significant variation of fluorescence between dilutions of the antibody in the coupling was observed, indicating that a test with smaller antibody values would be required in order to determine the best concentration for coupling and the limiting dilution.

\subsection{Magnetic studies}

Figure 9 shows the magnetization as a function of magnetic field of CARB $-\gamma \mathrm{Fe}_{2} \mathrm{O}_{3}$ and ATPS $-\gamma \mathrm{Fe}_{2} \mathrm{O}_{3}$ beads. At $300 \mathrm{~K}$, the CARB- $\gamma \mathrm{Fe}_{2} \mathrm{O}_{3}$ and ATPS- $\gamma \mathrm{Fe} 2 \mathrm{O} 3$ samples showed saturation magnetizations of $27.4 \mathrm{emu} \mathrm{g}^{-1}$ and $26.4 \mathrm{emu} \mathrm{g}^{-1}$, respectively. At $5 \mathrm{~K}$, their saturation magnetizations were of $35.5 \mathrm{emu} \mathrm{g}^{-1}$ and $33.9 \mathrm{emu} \mathrm{g}^{-1}$ for CARB $-\gamma \mathrm{Fe}_{2} \mathrm{O}_{3}$ and ATPS $-\gamma \mathrm{Fe}_{2} \mathrm{O}_{3}$ beads, respectively. The low saturation magnetization is due to the $\mathrm{SiO}_{2}$ contribution to the sample mass - as shown in figure 2, there is a thick layer of $\mathrm{SiO}_{2}$ on the surfaces of $-\gamma \mathrm{Fe}_{2} \mathrm{O}_{3} \mathrm{NPs}$ - and to the 
Table 1. Percentage of APTS- $\gamma \mathrm{Fe}_{2} \mathrm{O}_{3}$ and CARB $-\gamma \mathrm{Fe}_{2} \mathrm{O}_{3}$ beads coupled with antibodies separated into high coupling and low coupling.

\begin{tabular}{lccccc}
\hline \multirow{2}{*}{ Sample } & \multicolumn{3}{c}{ High $\%$} & & \multicolumn{2}{c}{ Low $\%$} \\
\cline { 2 - 3 } \cline { 5 - 6 } \cline { 5 - 6 } & $\mathrm{APTS}-\gamma \mathrm{Fe}_{2} \mathrm{O}_{3}$ & $\mathrm{CARB}-\gamma \mathrm{Fe}_{2} \mathrm{O}_{3}$ & & APTS- $\gamma \mathrm{Fe}_{2} \mathrm{O}_{3}$ & $\mathrm{CARB}-\gamma \mathrm{Fe}_{2} \mathrm{O}_{3}$ \\
\hline $\mathrm{CN}$ & 0 & 0.052 & & 0.37 & 2.57 \\
$10 \mu \mathrm{g}$ & 95.2 & 88.1 & & 4.62 & 10.4 \\
$8 \mu \mathrm{g}$ & 88.9 & 76.2 & & 11 & 2.7 \\
$5 \mu \mathrm{g}$ & 93.1 & 87 & & 6.8 & 10.6 \\
$2.5 \mu \mathrm{g}$ & 95.9 & 82.4 & & 3.92 & 15.9 \\
$2 \mu \mathrm{g}$ & 90.9 & 89 & & 8.94 & 9.32 \\
$1 \mu \mathrm{g}$ & 96.6 & 81.1 & & 3.29 & 14.2 \\
$0.5 \mu \mathrm{g}$ & 94.8 & 91 & & 4.89 & 7.86 \\
$\mathrm{Mean}$ & 93.3 & 85 & & 6.21 & 12.7 \\
$\mathrm{SD}$ & 2.83 & 5.24 & & 2.85 & 4.49 \\
\hline
\end{tabular}

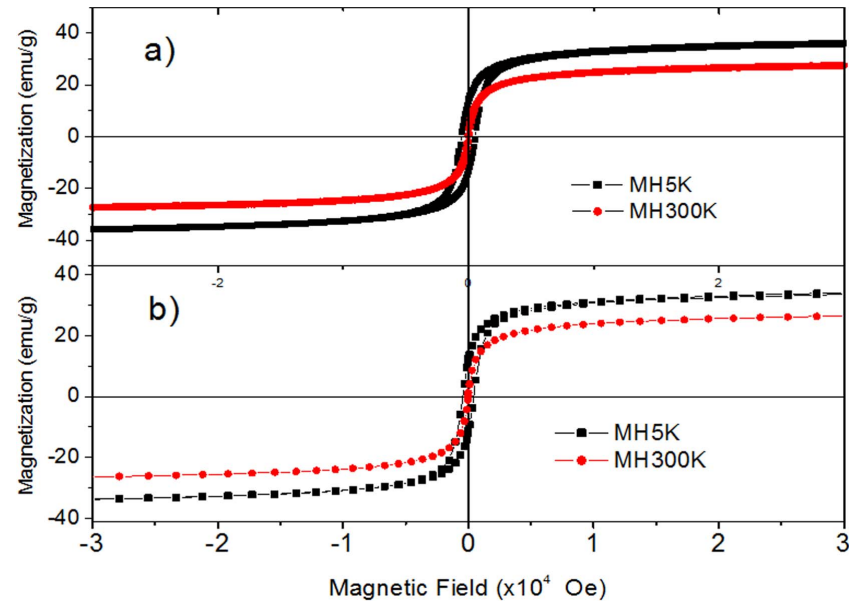

Figure 9. $\mathrm{M}-\mathrm{H}$ curves at $5 \mathrm{~K}$ and $300 \mathrm{~K}$ for (a) Carboxylic acid (CARB- $\gamma \mathrm{Fe}_{2} \mathrm{O}_{3}$ ) beads and (b) ATPS (ATPS- $\gamma \mathrm{Fe}_{2} \mathrm{O}_{3}$ ) beads.

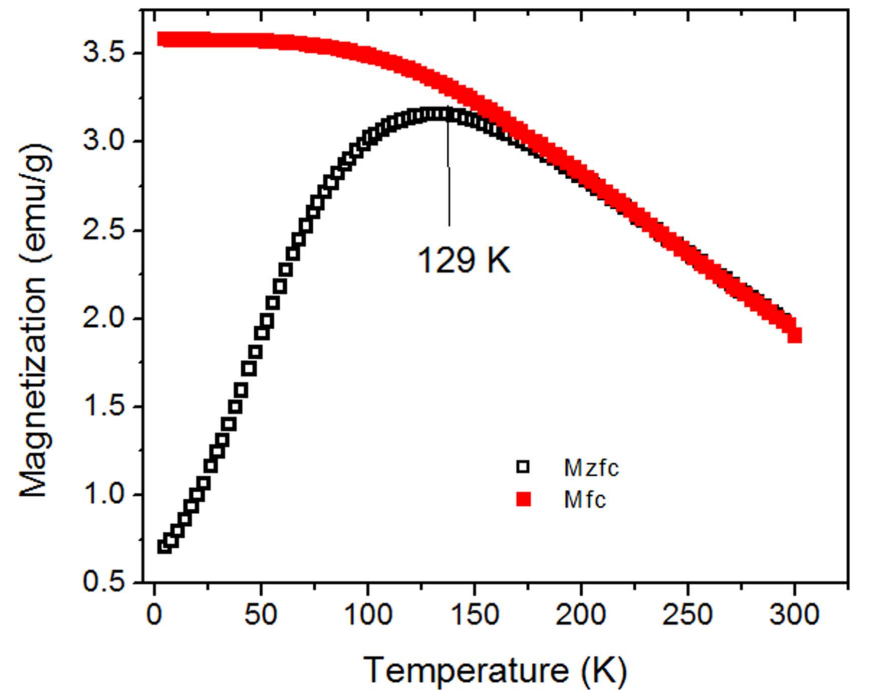

Figure 10. Mzfc and Mfc measurement under a field of 70 Oe for ATPS $-\gamma \mathrm{Fe}_{2} \mathrm{O}_{3}$ beads.

reduced $\gamma \mathrm{Fe}_{2} \mathrm{O}_{3}$ particle size, which enhances the total surface of the sample; in this case, the outer Fe layers have broken bonds and their Fe moments may experience a canting effect and therefore show a lower saturation magnetization [23].

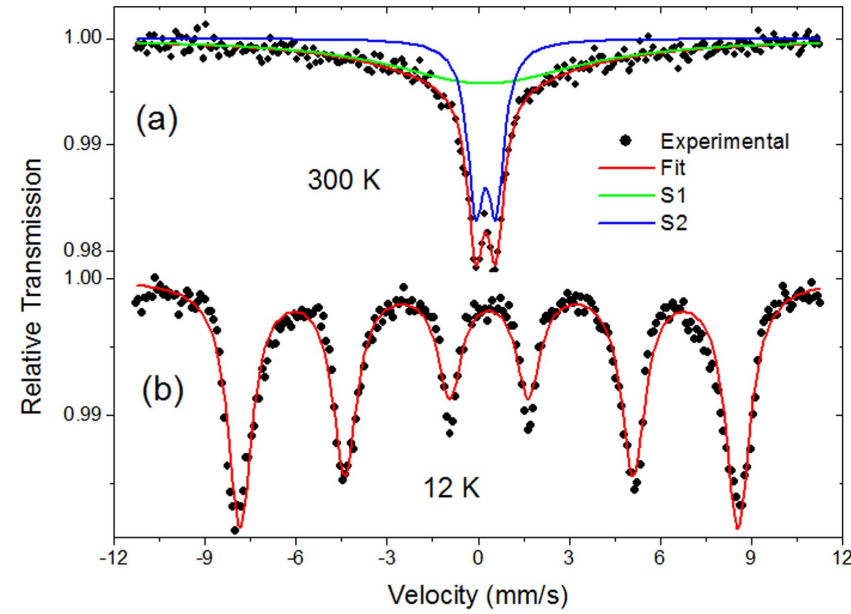

Figure 11. Mössbauer spectroscopy measurements performed at (a) $300 \mathrm{~K}$ and (b) $12 \mathrm{~K}$ for CARB- $\gamma \mathrm{Fe}_{2} \mathrm{O}_{3}$ beads.

At room temperature the coercivity field for CARB- $\gamma \mathrm{Fe}_{2} \mathrm{O}_{3}$ and ATPS- $\gamma \mathrm{Fe}_{2} \mathrm{O}_{3}$ samples were smaller than 50 Oe. Although the NPs are below the critical size to be superparamagnetic at room temperature, the low coercivity field suggest that there is some agglomeration of NPs and there are dipolar interactions between them.

Figure 10 shows the Mzfc and Mfc measurements performed for the ATPS $-\gamma \mathrm{Fe}_{2} \mathrm{O}_{3}$ sample. The cusp observed at $T_{B}{ }^{V S M}=129 \mathrm{~K}$ indicates the thermal blocking temperature for the ATPS- $\gamma \mathrm{Fe}_{2} \mathrm{O}_{3} \mathrm{NPs}$, this temperature may be larger than expected for a system of NPs without interaction [24]. The magnetic dipolar interaction between NPs can be studied through long-range dipolar forces in terms of the interacting superparamagnetic model and the Vogel-Fulcher law [24]. Since the CARB- $\gamma \mathrm{Fe}_{2} \mathrm{O}_{3}$ sample was obtained by functionalization of the ATPS $-\gamma \mathrm{Fe}_{2} \mathrm{O}_{3}$ sample with succinic acid, it is reasonable to conclude that both samples should have similar blocking temperatures.

The ${ }^{57} \mathrm{Fe}$ Mössbauer spectra for the CARB- $\gamma \mathrm{Fe}_{2} \mathrm{O}_{3}$ sample is presented in figure 11 . The spectrum recorded at room temperature, figure 11(a), is analyzed by taking into account a broad spectrum (S1) due to slow relaxing $\mathrm{Fe}$ magnetic moments, and a doublet (S2) related to fast relaxing Fe magnetic moments. The doublet has isomer shift and 

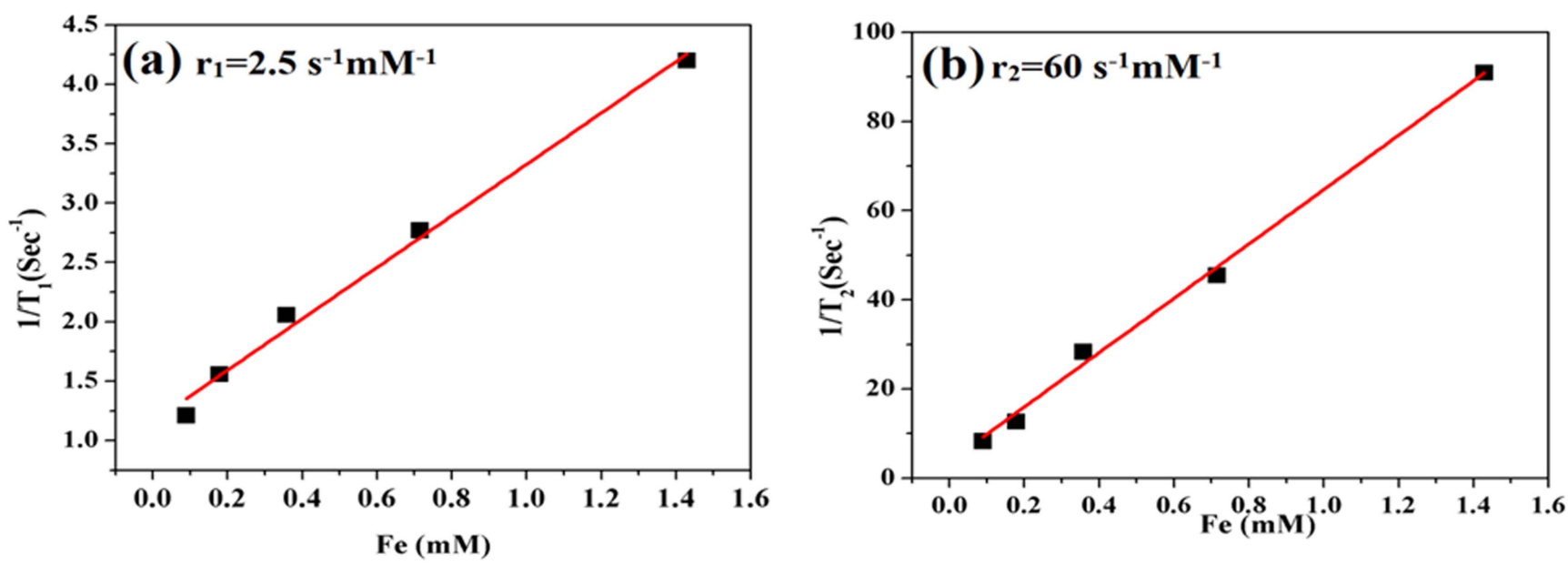

Figure 12. MRI relaxation time of the as-prepared beads $\mathrm{CARB}-\gamma \mathrm{Fe}_{2} \mathrm{O}_{3}$ at various concentrations of $\mathrm{Fe}(0.08,0.18,0.36,0.72$ and $1.43 \mathrm{mM})$.

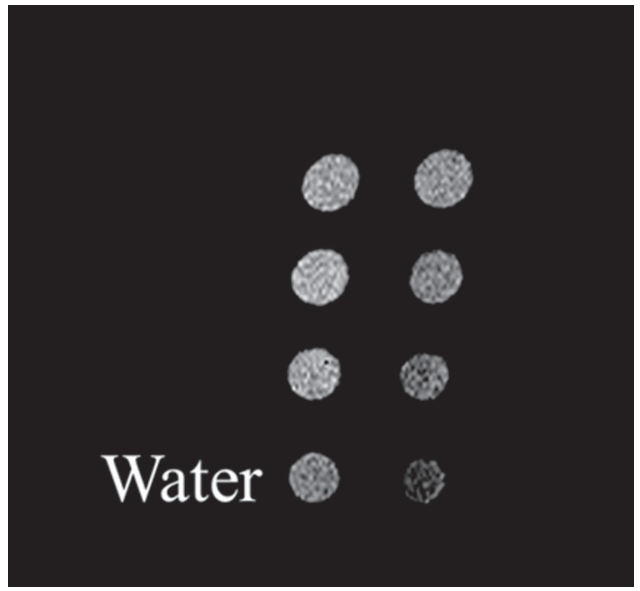

Figure 13. $\mathrm{T} 2 \mathrm{MR}$ imaging of pure water and $\mathrm{CARB}-\gamma \mathrm{Fe}_{2} \mathrm{O}_{3}$ beads at various concentrations of $\mathrm{Fe}(0.08,0.18,0.36,0.72,1.43,2.14$ and $2.85 \mathrm{mM}$ )

quadrupole splitting in agreement with $\mathrm{Fe}^{3+}$. The relative absorption spectral areas (RAA) are of $63 \%$ and $37 \%$ for the $\mathrm{S} 1$ and S2 Fe components. Since RAA(S1)/RAA(S2) $>1$, we can conclude that this sample is thermally blocked at $300 \mathrm{~K}$. This result does not contradict the $\mathrm{M}-\mathrm{H}$ analyses because the Mössbauer spectroscopy measuring time (of $\sim 10^{-7} \mathrm{~s}$ ) is smaller than the magnetometry measuring time $(\sim 10 \mathrm{~s})$; this fact will lead to a higher Mössbauer blocking temperature.

${ }^{57} \mathrm{Fe}$ Mössbauer spectroscopy is an element-specific technique which provides local information on the spin relaxation and charge of iron ions, and allows an easy identification of iron oxides through their hyperfine magnetic field, isomer shift and quadrupole splitting [25].

The measurement recorded at $12 \mathrm{~K}$ is shown in figure 11(b). This spectrum exhibits a single sextet with hyperfine magnetic field of $51 \mathrm{~T}$ and isomer shift of $0.38 \mathrm{~mm} \mathrm{~s}^{-1}$; both parameters are in close agreement with the formation of maghemite [25]. No other second phase containing Fe was observed.

\subsection{Magnetic resonance studies}

Interest in the use of nanometric particles has increased over recent years, and has been applied in several studies. Many studies have sought to characterize and investigate applications, even proposing the use of the tool as a diagnostic test material due to its easy applicability. Flow cytometry has been used as a tool to evaluate the morphometric characteristics [26] and coupling capacity [27] of these compounds, as well as the interaction which these NPs can have with human cells, increasing the applicability of these compounds in the field of immunology [27-30]. In our study, beads prepared with APTS- $\gamma \mathrm{Fe}_{2} \mathrm{O}_{3}$ and CARB- $\gamma \mathrm{Fe}_{2} \mathrm{O}_{3}$ showed similar morphologies (figure 6) as well as sample uniformity (figure 7). The ability to investigate these morphological parameters and the coupling capacity of these NPs analyzed by flow cytometry may be fundamental for the development of a future tool for applications in nanobiotechnology [31-33].

In order to examine the features of as-synthesized beads CARB- $\gamma \mathrm{Fe}_{2} \mathrm{O}_{3}$ as an MR contrast agent, magnetic relaxation times and $T_{2}$ magnetic resonance images were measured on $0.55 \mathrm{~T}$ scanner.

The longitudinal and transverse relaxation times $\left(r_{2}, r_{1}\right)$ of CARB- $\gamma \mathrm{Fe}_{2} \mathrm{O}_{3}$ beads are shown in figures 12(a), (b). The $r_{1}$ value is $2.5 \mathrm{~s}^{-1} \mathrm{mM}^{-1}$ and the $r_{2}$ value $60 \mathrm{~s}^{-1} \mathrm{mM}^{-1}$, giving an $r_{2} / r_{1}$ ratio of 24- demonstrating that the fabricated beads are an efficient $T_{2}$ weighted contrast agent.

Furthermore, to prove the relation time in the favor of $T_{2}$ contrast agent, MR imaging was acquired. Figure 13 shows the $T_{2}$-weighted MRI of beads and water. It is clearly seen that the MRI signals of NPs becomes brighter at initial low concentration because of the size of the NPs and then become darkened at high concentration as compared to the MRI image of pure water. The MRI shows that imaging intensity increases with the increase of $\mathrm{Fe}$ concentration. The MR relaxation data and MRI results showed the potential of assynthesized NPs being used as $T_{2}$ MRI contrast agent. 


\section{Conclusions}

Magnetic $\gamma \mathrm{Fe}_{2} \mathrm{O}_{3}$ NPs with mean size $6.5 \mathrm{~nm}$ were successfully synthesized by a sonochemical method. Polycondensation of APTS on $\gamma \mathrm{Fe}_{2} \mathrm{O}_{3}$ NPs led to the formation of APTS $-\gamma \mathrm{Fe}_{2} \mathrm{O}_{3}$ beads. These beads were modified with succinic anhydride to obtain CARB- $\gamma \mathrm{Fe}_{2} \mathrm{O}_{3}$ beads. The size of the beads was evaluated by TEM, which returned a mean size of $12 \mathrm{~nm}$. The DLS results showed that the beads were aggregated and have a hydrodynamic size of $490 \mathrm{~nm}$. The $\mathrm{CARB}-\gamma \mathrm{Fe}_{2} \mathrm{O}_{3}$ and APTS- $\gamma \mathrm{Fe}_{2} \mathrm{O}_{3}$ beads had isoelectric points at $\mathrm{pH}$ of 3.76 and 6.41 , respectively. The FTIR studies showed characteristic bands which demonstrated the presence of the amino and silane groups, at $870 \mathrm{~cm}^{-1}$ and $1352 \mathrm{~cm}^{-1}$, respectively, as well as carboxylate groups, at $1648 \mathrm{~cm}^{-1}$ and $1425 \mathrm{~cm}^{-1}$. To prove the ability of magnetic beads to couple proteins, rabbit antibody was covalently immobilized on these chemically modified beads. Mössbauer spectroscopy and VSM measurements showed that a large fraction of the beads have superparamagnetic behavior within the VSM measuring time. The XRD and Mössbauer results showed that capping with APTS did not induce any phase change in the maghemite phase. Immobilization time of rabbit antibodies was $4 \mathrm{~h}$ at a concentration of $120 \mathrm{mg}$ per gram of NPs. The MRI results suggest that magnetic beads indeed constitute an excellent $T_{2}$ contrast agent candidate.

\section{Acknowledgments}

Authors thank the financial support of the Brazilian agencies $\mathrm{MCT} / \mathrm{CNPq}$, CAPES and INCT in Nanobiotechnology. Morales $\mathrm{M}$ thanks CNPq for his fellowship.

\section{ORCID iDs}

A F R Rodriguez (ii) https://orcid.org/0000-0002-3034-183X

\section{References}

[1] Dyal A, Loos K, Noto M, Chang S W, Spagnoli C, Shafi K V P M, Ulman A, Cowman M and Gross R A 2003 Activity of candida rugosa lipase immobilized on $\gamma-\mathrm{Fe}_{2} \mathrm{O}_{3}$ magnetic nanoparticles J. Am. Chem. Soc. 125 1684-5

[2] Yang H, Zhang S, Chen X, Zhuang Z, Xu J and Wang X 2004 Magnetite-containing spherical silica nanoparticles for biocatalysis and bioseparations Anal. Chem. 76 1316-21

[3] Lee Y, Lee H, Kim Y B, Kim J, Hyeon T, Park H W, Messersmith P B and Park T G 2008 Bioinspired surface immobilization of hyaluronic acid on monodisperse magnetite nanocrystals for targeted cancer imaging $A d v$. Mater. 20 4154-7

[4] Aphesteguy J C, Kurlyandskaya G V, de Celis J P, Safronov A P and Schegoleva N N 2015 Magnetite nanoparticles prepared by co-precipitation method in different conditions Mater. Chem. Phys. $161243-9$

[5] Xu J, Yang H, Fu W, Du K, Sui Y, Chen J, Zeng Y, Li M and Zou G 2007 Preparation and magnetic properties of magnetite nanoparticles by sol-gel method J. Magn. Magn. Mater. 309 307-11

[6] Karami H 2010 Synthesis and characterization of iron oxide nanoparticles by solid state chemical reaction method J. Clust. Sci. 21 11-20

[7] Kim E H, Lee H S, Kwak B K and Kim B-K 2005 Synthesis of ferrofluid with magnetic nanoparticles s by sonochemical method for MRI contrast agent J. Magn. Magn. Mater. 289 328-30

[8] Li C, Wei Y, Liivat A, Zhu Y and Zhu J 2013 Microwavesolvothermal synthesis of $\mathrm{Fe}_{3} \mathrm{O}_{4}$ magnetic nanoparticles Mater. Lett. 107 23-6

[9] Unni M, Uhl A M, Savliwala S, Savitzky B H, Dhavalikar R, Garraud N, Arnold D P, Kourkoutis L F, Andrew J S and Rinaldi C 2017 Thermal decomposition synthesis of iron oxide nanoparticles with diminished magnetic dead layer by controlled addition of oxygen ACS Nano 11 2284-303

[10] Berry C C 2000 Progress in functionalization of magnetic nanoparticles for applications in biomedicine $J$. Phys. D. Appl. Phys. 42224003

[11] Hankanes B I and Kvam C 1993 Application of magnetic beads in bioassay Bio/Technology 11 60-3

[12] Hai N H, Luong N H and Chau N 2009 Preparation of magnetic nanoparticles embedded in polystyrene microspheres J. Phys.: Conf. Ser. 187012009

[13] Morimoto Y, Abe M, Hatakayama M, Handa H and Sandhu A 2009 Detection of magnetic nanobeads by self-assembly of superparamagnetic microbeads for biosensing IEEE Trans. Magn. 45 2871-4

[14] Bruce I J and Sen T 2005 Surface modification of magnetic nanoparticles with alkoxy-silanes and their application in magnetic bioseparations Langmuir 21 7029-35

[15] Xie J, Liu G, Eden H S, Ai H and Chen X 2011 Surfaceengineered magnetic nanoparticle platforms for cancer imaging and therapy Acc. Chem. Res. 44 883-92

[16] Jain T K, Morales M A, Sahoo S K, Leslie-Pelecky D L and Labhasetwar V 2005 Iron oxide nanoparticles for sustained delivery of anticancer agents Mol. Pharm. 2 194-205

[17] Kopp W, Costa T, Pereira S C, Jafelicci M, Giordano R C, Marques R F C, Araujo-Moreira F M and Giordano R L C 2014 Easily handling penicillin $\mathrm{G}$ acylase magnetic crosslinked enzymes aggregates: catalytic and morphological studies Process Biochem. 49 38-46

[18] Guo H, Yu Y, Xue L and Quian J 2016 Covalent immobilization of $\alpha$-amylase on magnetic particles as catalyst for hydrolysis of high-amylose starch Int. J. Biol. Macromolecules 287 537-44

[19] Greg T H 2013 Bioconjugate Techniques (New York: Academic)

[20] Socrates G 1994 Infrared Characteristic Group Frequencies: Tables and Charts (New York: Wiley)

[21] Nakamoto K 1970 Infrared Spectra of Inorganic and Coordination Compounds (New York: Wiley)

[22] Yu S and Chowa G M 2004 Carboxyl group $\left(-\mathrm{CO}_{2} \mathrm{H}\right)$ functionalized ferrimagnetic iron oxide Nanoparticles for potential bio-applications J. Mater. Chem. 14 2781-6

[23] Tronc E et al 2003 Surface effects in noninteracting and interacting $\gamma-\mathrm{Fe}_{2} \mathrm{O}_{3}$ nanoparticles J. Magn. Magn. Mater. 262 6-14

[24] Moscoso-Londoño O et al 2017 Different approaches to analyze the dipolar interaction effects on diluted and concentrated granular superparamagnetic systems J. Magn. Magn. Mater. 428 105-18

[25] Cornell R M and Schwertmann U 1996 The Iron Oxides (New York: Wiley) Ch 6

[26] Verschoor C P, Lelic A, Bramson J L and Bowdish D M E 2015 An introduction to automated flow cytometry gating tools and their implementation Frontiers Immunol. 6 1-9

[27] Neurauter A A, Bonyhadi M, Lien E, Nokleby L, Ruud E, Camacho S and Aavark T 2007 Cell isolation and expansion 
using dynabeads cell separation Advances Biochem. Eng./ Biotechnol. 106 41-73

[28] Zahavy E, Ber R, Gur D, Abramovich H, Freeman E, Maoz S and Yitzhaki S 2012 Application of nanoparticles for the detection and sorting of pathogenic bacteria by flowcytometry Advances Exp. Med. Biol. 733 23-36

[29] Zahavy E, Heleg-Shabtai V, Zafrani Y, Marciano D and Yitzhaki S 2010 Application of fluorescent nanocrystals (q-dots) for the detection of pathogenic bacteria by flowcytometry J. Fluorescence 20 389-99

[30] Cai D et al 2008 Interaction between carbon nanotubes and mammalian cells: characterization by flow cytometry and application Nanotechnology 19345102
[31] Hu H, Hou X-J, Wang X-C, Nie J-J, Cai Q and Xu F-J 2016 Gold nanoparticle-conjugated heterogeneous polymer brushwrapped cellulose nanocrystals prepared by combining different controllable polymerization techniques for theranostic applications Polym. Chem. 7 3107-16

[32] Xianbin Y et al 2003 Immunofluorescence assay and flowcytometry selection of bead-bound aptamers Nucleic Acids Res. 31 e54

[33] van der Vlist E J, Hoen H E N, Stoorvogel W, Arkesteijn G J and Wauben M H 2012 Fluorescent labeling of nano-sized vesicles released by cells and subsequent quantitative and qualitative analysis by high-resolution flow cytometry Nat. Protocols 7 1311-26 